\title{
Design and Analysis of Flexible Hinge Used for Unfolding Spacecraft Solar Panels
}

\author{
Jing Zhang1,*, Kai Yan¹, Ziming Kou ${ }^{1}$
}

\author{
Zhang J (iD https: / /orcid.org/0000-0001-5735-8132 \\ Yan K (iD https://orcid.org/0000-0003-2920-793X \\ Kou Z (D) https://orcid.org/0000-0001-9260-612X
}

\author{
How to cite \\ Zhang J; Yan K; Kou Z (2019) Design and Analysis of Flexible \\ Hinge Used for Unfolding Spacecraft Solar Panels. J Aerosp \\ Technol Manag, 11: e3319. https://doi.org/10.5028/ \\ jatm.v11.1060
}

ABSTRACT: Two stiffness models for a flexible hinge with large rotation angle are established based on the pseudo-rigid body method and the series or parallel relationship of flexible units. Finite element simulation of the flexible hinge is conducted in ANSYS to verify the two stiffness models of the flexible hinge. A multi-objective optimization method is used to optimize the design parameters of the hinge. The stiffness models of the flexible hinge are used to establish the optimized objective function of an optimization model to improve the rotation angle of the hinge under a certain radial stiffness. After optimization, the rotation angle can reach 70 deg when the rotational and radial stiffnesses are $1.29 \mathrm{~N} \cdot \mathrm{mm} / \mathrm{rad}$ and $1.37 \mathrm{~N} / \mathrm{mm}$.

KEYWORDS: Flexible hinge, Equivalent stiffness, Multi-objective optimization, Constrained optimization.

\section{INTRODUCTION}

The solar sail, which is generally formed by articulating several solar panels, is the main source of energy for the spacecraft in orbit. It plays an important role in the normal work of the spacecraft (Fenci and Currie 2017; Meguro et al. 2009; McGuire et al. 2016). The gaps and frictions in the traditional hinges can cause vibration, which is bad for the control of space status (Li et al. 2016; Sun and Chen 2016). However, one advantage of flexible hinges without gap, friction, and lubrication, is integrated processing. Flexible mechanism as a new type of mechanisms uses the elastic deformation of material to transfer motion, force and energy (Delimont et al. 2015; Dewalque et al. 2016). To realize the deployment of space structures, the flexible hinge should rotate in large rotational angle. The usually studied hinges have a small allowable rotational angle or a large volume. The hinges are hard to realize the deployment of structures. Therefore, designing a hinge with a large rotational angle and a small volume is important.

Four classes of the design methods used for the compliant structure design are freedom and constraint topology (FACT), the screw theory, type synthesis, and a building block approach (Hopkins and Culpepper 2011; Sun and Hopkins 2017; Hopkins and Panas 2013; Su and Yue 2013; Naves et al. 2017). Elliptic integral methods and pseudo-rigid-body methods are the main methods for the stiffness calculation of flexible hinges (Liu and Yan 2017; Midha et al. 2015; Šalinić and Nikolić 2018; Howell 2001; Qiu et al. 2014). Nonlinear multi-objective constrained optimization can be conducted by using the constraint majorized function of MATLAB.

In this study, a kind of flexible hinge is designed based on the constraint analysis of plane and line. Two stiffness models of hinge are established in the third section to express the relationship of the out-of-plane and in-plane deformations with the load on the hinge. The stiffness models can also be used to build the optimization model. Finite element simulation is conducted to verify the stiffness model in the fourth section. In the fifth section, the optimization is conducted to optimize the size of the hinge.

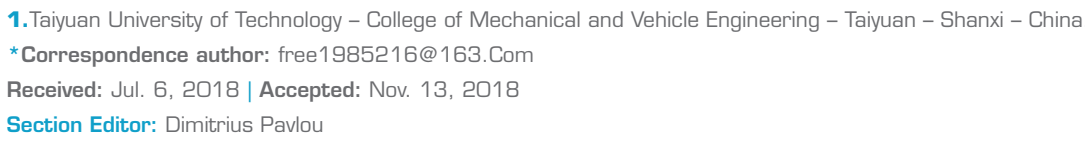




\section{FLEXIBLE HINGE DESIGN BASED ON CONSTRAINT ANALYSIS}

A constraint plane, which limits two translational degrees of freedom and one rotational degree of freedom in one plane, can be considered as three constraint lines (two parallel lines and one intersecting line) in the same plane. The five constraint lines can be replaced by five bars. The five bars, which are located in two planes, are equal to two constraint planes. As shown in Fig. 1, the constraint plane 1 can be replaced by three bar units $\mathrm{B}_{1}, \mathrm{~B}_{2}$, and $\mathrm{B}_{5}$, which restrict the translations in the $x$ and $z$ directions, and the rotation in $x z$ plane. The constraint plane 2 can be replaced by bar units $\mathrm{B}_{3}, \mathrm{~B}_{4}$, and $\mathrm{B}_{6}$. The bar units limit the translations in the $y$ and $z$ directions, and the rotation in $x z$ plane. There is a redundant constraint corresponding to the translation in $z$ direction. Therefore, these two planes limit five degrees of freedom.

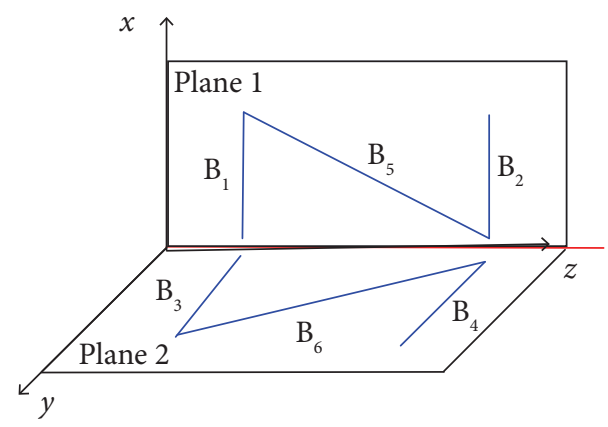

Figure 1. Plate-constraint of flexible hinge.

There are two kinds of spatial locations for two planes, parallel and intersection. The two constraint planes can be replaced by two plates. The plate constraint can be divided by the spatial location and the deformed form. The deformation types of the plates can be bend or torsion.

Based on the constraint analysis, two intersecting plates can be enough to limit the five degrees of freedom and achieve a rotational motion. Three plates uniformly distributed in the rotational plane are adopted in the flexible hinge to increase the stability. In order to design a flexible hinge with characteristics of large rotation angle and high radial stiffness, the bending and torsional deformations and the series and parallel relationships are included in a hinge. A hinge includes six flexible units, shown in Fig. 2. The flexible hinge consists of three parts, one inner cylinder, one outer ring, and six flexible units. Two flexible units in one plane are defined as one group. A flexible unit is composed of five straight beams connected in a series and numbered as 1 to 5. The series beams can enlarge the allowable rotation angle of the hinge. The radii of the inner cylinder and outer ring are $r$ and $R$, respectively. The lengths of the beams 1,3 , and 5 are $L_{1}, L_{3}$, and $L_{5}$, respectively. The lengths of the beams 2 and 4 are $L_{2}$ and $L_{4}$, respectively. The width and thickness are $b$ and $h$, respectively. In this design, $L_{2}=L_{4}, L_{1}=L_{5}, L_{3}=L_{1}+L_{5}+r-R$.
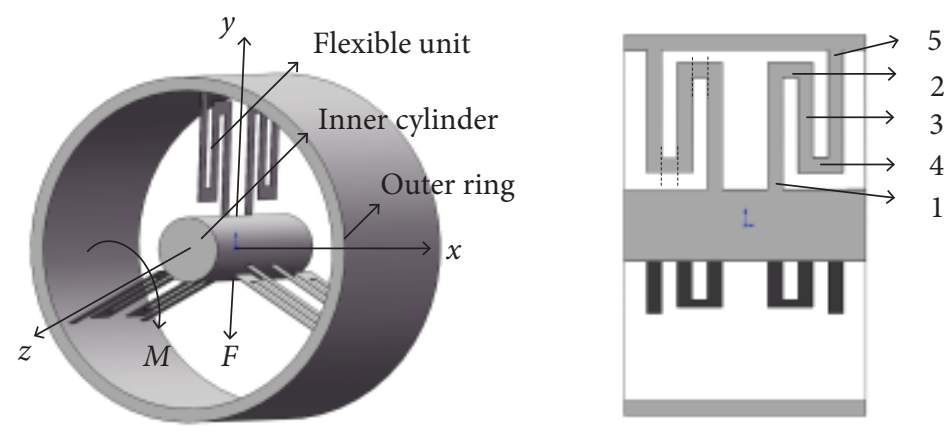

Figure 2. The structure of flexure hinge. 


\section{STIFFNESS MODELING}

\section{THEORETICAL BASIS}

\section{Pseudo-Rigid Body Method}

In the pseudo-rigid body method the flexible structures are replaced by torsional springs and bars. Then, a compliant mechanism can be defined as a mechanism consisting of rigid bodies. The relationship of the force and deformation of the mechanism with rigid bodies is similar to the compliant mechanism. When the flexible hinge is under a $z$-direction torque, the flexible unit in the flexible hinge can be replaced by the fixed-guide beam unit, which is one type of structure in the pseudo-rigid body method (Liu and Yan 2017; Midha et al. 2015). The model is shown in Fig. 3.
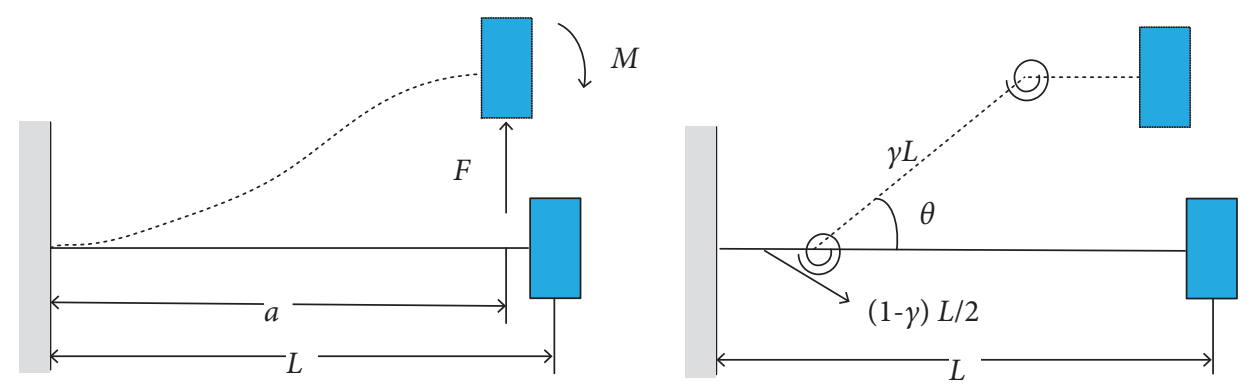

Figure 3. The deformation of fixed-oriented beam.

In Fig. 3, $F$ and $M$ are the force and moment applied on the end of the beam. The length of the beam is expressed by $L$. The horizontal length of the deformed beam is $a$. The characteristic radius coefficient of the pseudo-rigid body model of the fixed-guide beam is $\gamma$. The rotation angle in the fixed-guide beam model is $\theta$. Because the anti-symmetry of the fixed-guide beam deformation about the midpoint exists, the force and moment satisfy $M=F a / 2$. Stiffness constant of spring can be given as (Eq. 1) (Howell 2001):

$$
K_{\mathrm{t}}=2 \gamma k_{\theta} \frac{E I}{L}
$$

where: $\gamma=0.85 ; k_{\theta}$ is the stiffness coefficient, $k_{\theta}=2.65 ; E$ is elastic modulus; and moment of inertia $I$ can be given as $I=b h^{3} / 12$.

\section{Series-Parallel Connection of Flexible Unit}

One flexible unit contains five beams, shown in Fig. 2. Each beam can be equivalent to a spring. The stiffnesses of beams 1 to 5 are expressed as $K_{1}, K_{2}, K_{3}, K_{4}$, and $K_{5}$, respectively. The relationship between the total stiffness and the stiffness of each beam is given in Fig. 4. According to the parallel relationship of springs, the stiffnesses of the six flexible units of the flexible hinge can be expressed as $K_{I} \sim K_{V I}$, where $K_{I}=\sim=K_{V I}$.

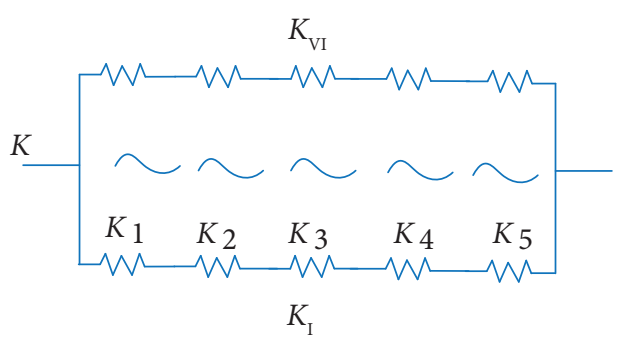

Figure 4. The equivalent stiffness of flexure hinge. 
Based on the series relationship, the stiffness of one flexible unit is given as (Eq. 2)

$$
K_{i}=\frac{1}{\sum_{j=1}^{5} \frac{1}{K_{j}}}(i=\mathrm{I} \sim \mathrm{VI})
$$

The overall stiffness of the hinge $K$ can be derived from the parallel relationship of the springs (Eq. 3 )

$$
K=6 K_{\mathrm{I}}
$$

\section{Rotational Stiffness of Flexible Hinge}

When torque is applied on the inner cylinder in $z$ direction, the inner cylinder rotates on its own axis. Due to the symmetry of the structure, the stiffness of one flexible unit can be calculated to get the stiffness of the hinge. The deformation type of the five beams in one flexible unit can be divided into two types, bend and torsion. Because the size of the beams 1 and 5 are equal, and the beams 2 and 4 are equal, the relationships between the stiffnesses satisfy (Eq. 4)

$$
K_{1}=K_{5}, \quad K_{2}=K_{4}
$$

\section{BENDING BEAM}

In Fig. 5, when the rotation angle of pseudo-rigid body is $\theta_{1}$, the rotation angle of the end of the beam 1 relative to the inner cylinder is $\alpha_{1}$. The arc length, which corresponds to the angle $\theta_{1}$ in pseudo-rigid body, is $s_{1}$. The arc length, which corresponds to the angle $\alpha_{1}$, is $s_{2}$. Torque applied on the beam 1 is $M_{1}^{\mathrm{B}}$, the rotational stiffness of beam 1 in pseudo-rigid body can be given as (Eq. 5)

$$
K^{\mathrm{B} 1}=\frac{M_{1}^{\mathrm{B}}}{\theta_{1}}
$$

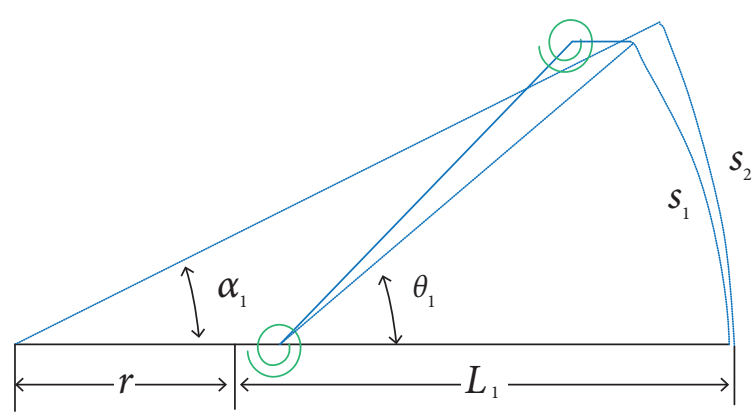

Figure 5. The Pseudo-rigid-body Model of Beam 1.

The geometry function of the relationship between $s_{1}$ and $s_{2}$ is established. The difference between $s_{1}$ and $s_{2}$ is calculated when $\alpha_{1}$ changes from $1^{\circ}$ to $90^{\circ}$. The difference increases with the angle $\alpha_{1}$. The minimum difference is $12 \%$, when $\alpha_{1}=1^{\circ}$. The maximum difference is $19 \%$, when $\alpha_{1}=90^{\circ}$. Therefore, in order to simplify the geometrical relationship, it is assumed that the arc $s_{2}$ corresponding to $\alpha_{1}$ is equal to the $\operatorname{arc} s_{1}$. Therefore, the relationship of $\theta_{1}$ and $\alpha_{1}$ can be given as (Eq. 6)

$$
\theta_{1} \gamma L_{1}=\alpha_{1} R
$$


The stiffness constant of the spring in beam 1 can also be expressed as $K^{\mathrm{B} 1}=2 \gamma k_{\theta} E I_{1} / L_{1}$, where the inertia moment of beam 1 is $I_{1}=I=b h^{3} / 12$. Based on the Eqs. 5 and 6, the bending stiffness of the beam 1 in the hinge is (Eq. 7)

$$
K_{1}^{\mathrm{B}}=\frac{M_{1}^{\mathrm{B}}}{\alpha_{1}}=\frac{k_{\theta} R E b h^{3}}{6 L_{1}{ }^{2}}
$$

Based on the same deducing, the bending stiffness of the beams 1, 3, and 5 can be given as (Eq. 8)

$$
K_{i}^{\mathrm{B}}=\frac{k_{\theta} R E b h^{3}}{6 L_{i}^{2}}(i=1,3,5)
$$

\section{TWIST BEAM}

The stiffnesses of twist beams 2 and 4 in flexible unit have been given in mechanics of materials. The stiffness of beam 2 can be written as

$$
K^{\mathrm{T} 2}=\frac{b h^{3} G}{L_{2}}\left(\frac{1}{3}-0.21 \frac{h}{b}\right)
$$

where: $G$ is the shear modulus, $G=E /(2(1+\mu))$. The Poisson's ratio of the material is $\mu$.

When the twist beams deformed, the deformation diagram of a flexible unit is shown in Fig. 6 . The twist angles of the torsion beams 2 and 4 are equal to $\theta_{2}$, which correspond to the arc length $s_{3}$. The angle of the outer ring in relation to the inner cylinder is $\alpha_{2}$, which corresponds to the arc length $s_{4}$.

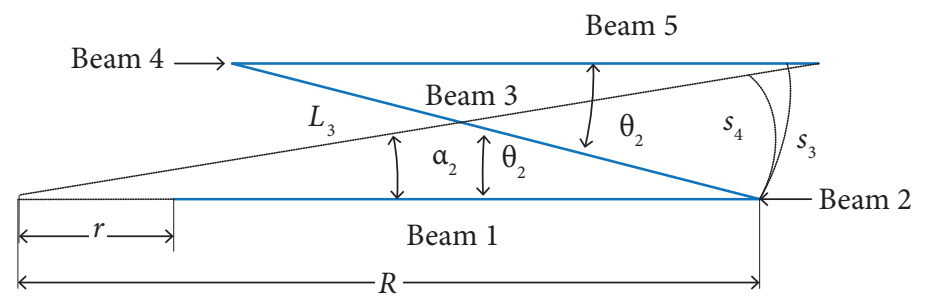

Figure 6. The corresponding arc and angle diagram of the torsion beam.

Because $L_{1}$ is close to the distance of the outer ring from the inner cylinder, the angles $\theta_{2}$ and $\alpha_{2}$ are close to each other when the rotation of the hinge is caused by the torsion of the beams 2 and 4 . Since the arc lengths of $\theta_{2}$ and $\alpha_{2}$ are approximately equal, the angles satisfy (Eq. 10)

$$
\theta_{2} L_{3}=\alpha_{2} R
$$

Based on Eqs. 9 and 10, the torsional stiffness of beams 2 and 4 can be given as (Eq. 11)

$$
K^{\mathrm{T}}=\frac{M_{\mathrm{a}}}{\alpha_{2}}=\frac{G R b h^{3}}{L_{2} L_{3}}\left(\frac{1}{3}-0.21 \frac{h}{b}\right)
$$

Considering the series relationship of the deformed beams, the rotational stiffness of one unit of the hinge can be given as (Eq. 12)

$$
K_{\mathrm{I}}=\left(\frac{1}{K_{1}^{\mathrm{B}}}+\frac{1}{K_{3}^{\mathrm{B}}}+\frac{1}{K_{5}^{\mathrm{B}}}+\frac{1}{K^{\mathrm{T}}}\right)^{-1}
$$


Based on the parallel relationship of the beams, the bending stiffness of flexible hinge can be given as (Eq. 13)

$$
K_{\mathrm{R}}=6 K_{\mathrm{I}}
$$

The rotational stiffness of the hinge is given as (Eq. 14)

$$
K_{\mathrm{R}}=\frac{6}{\frac{12\left(L_{1}{ }^{2}+\left(2 L_{1}+r-R\right)^{2}\right)}{k R E b h^{3}}+\frac{\left(2 L_{1}+r-R\right) L_{2}}{G R b h^{3}\left(\frac{1}{3}-0.21 \frac{h}{b}\right)}}
$$

\section{RADIAL STIFFNESS OF FLEXIBLE HINGE}

When force $F$ in $y$ direction is applied on the inner cylinder of the flexible hinge, the inner cylinder will move in $y$ direction. The radial stiffness of flexible hinges is expressed as $K_{\mathrm{T}}$.

\section{Strain Energy Calculation}

The directions of the three groups of flexible units are defined as 1,2 and 3. Every group contains two flexible units. The definitions of one flexible unit are shown in Fig. 7a. The half of the flexible unit I is shown in Fig. $7 \mathrm{~b}$.
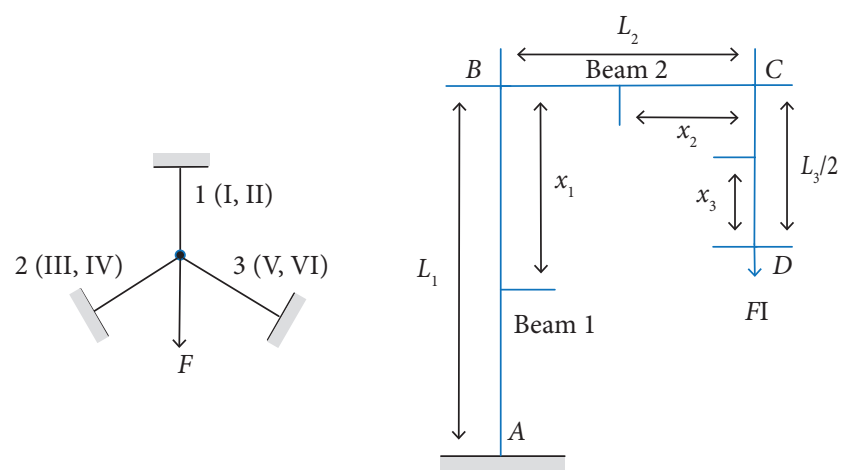

Figure 7. The definition and the force diagram. (a) Load and definition of flexible unit; (b) half of flexible unit I.

Based on the static indeterminacy theory, the force in each group can be given as $F_{1}=2 F_{2}=2 F_{3}=2 / 3 F$, where $F_{1}, F_{2}$, and $F_{3}$ are the forces in groups 1, 2, and 3. Because the structure shown in Fig. $7 \mathrm{~b}$ is symmetrical with respect to the centre of point $\mathrm{D}$, the stiffness of the half of the flexible unit is calculated. The half of the unit consists of three sections, AB, BC and CD. The total displacement energy of the deformed beam is given as (Eq. 15)

$$
V_{\varepsilon}=V_{\varepsilon}^{\mathrm{AB}}+V_{\varepsilon}^{\mathrm{BC}}+V_{\varepsilon}^{\mathrm{CD}}
$$

The internal force equation of the three-section can be given. In section CD (Eq. 16),

$$
M_{\mathrm{CD}}^{\mathrm{I}}\left(x_{3}\right)=0, F_{\mathrm{CD}}^{\mathrm{I}}\left(x_{3}\right)=F^{\mathrm{I}}
$$

In section $\mathrm{BC}$ (Eq. 17),

$$
M_{\mathrm{BC}}^{\mathrm{I}}\left(x_{2}\right)=F^{\mathrm{I}} x_{2}, F_{\mathrm{BC}}^{\mathrm{I}}\left(x_{2}\right)=0
$$


In section $\mathrm{AB}$ (Eq. 18),

$$
M_{\mathrm{AB}}^{\mathrm{I}}\left(x_{1}\right)=F^{\mathrm{I}} L_{2}, F_{\mathrm{AB}}^{\mathrm{I}}\left(x_{1}\right)=F^{\mathrm{I}}
$$

The entire deformation energy of one half of one flexible unit is given as (Eq. 19)

$$
\begin{aligned}
& +\int_{0}^{L_{1}} \frac{\left(F_{\mathrm{AB}}^{\mathrm{I}}\left(x_{1}\right)\right)^{2} \mathrm{~d} x_{1}}{2 E A}+\int_{0}^{L_{1}} \frac{\left(M_{\mathrm{AB}}^{\mathrm{I}}\left(x_{1}\right)\right)^{2} \mathrm{~d} x_{1}}{2 E I} \\
& =\left(F^{\mathrm{I}}\right)^{2}\left(\frac{L_{3}}{4 E A}+\frac{L_{2}^{3}}{6 E I}+\frac{L_{2}^{2} L_{1}}{2 E I}+\frac{L_{1}}{2 E A}\right)
\end{aligned}
$$

\section{The Stiffness Calculation of Single Flexible Unit}

The vertical displacement of point $\mathrm{D}$ is $\varepsilon$ when force $F^{\mathrm{I}}$ is applied on the point $\mathrm{D}$. The elastic strain energy caused by force $F^{\mathrm{I}}$ is written as (Eq. 20)

$$
W=\frac{1}{2} F^{\mathrm{I}} \varepsilon
$$

Because the elastic strain energy caused by force $F^{\mathrm{I}}$ is equal to the deformation energy, $F^{\mathrm{I}} \mathcal{\varepsilon} / 2=V$. The total deformation energy of the unit $\mathrm{I}$ is $F^{\mathrm{I}} \mathcal{\varepsilon}$. The radial stiffness of the flexible unit I is (Eq. 21)

$$
K^{\mathrm{I}}=\frac{F^{\mathrm{I}}}{2 \varepsilon}=\frac{1}{2} \frac{E b h}{\left(L_{1}+\frac{L_{3}}{2}\right)+\frac{12}{b^{2}}\left(L_{2}{ }^{2} L_{1}+\frac{L_{2}^{3}}{3}\right)}
$$

\section{Total Radial Stiffness Calculation}

Since there are two flexible units in one group, based on the force balance, the force satisfies $F^{\mathrm{I}}=F_{1} / 2=F / 3$. Therefore, the radial stiffness of the flexible hinge can be expressed as (Eq. 22)

$$
K_{\mathrm{T}}=3 K^{\mathrm{I}}=\frac{3 E b h}{2\left(\left(L_{1}+\frac{1}{2}\left(2 L_{1}+r-R\right)\right)+\frac{12 L_{2}^{2}}{b^{2}}\left(L_{1}+L_{2} / 3\right)\right)}
$$

\section{FINITE ELEMENT SIMULATIONS}

In order to verify the accuracy of the stiffness model, the analysis of the stiffness of the flexible hinge was conducted by using software ANSYS. The material of flexible hinge is Polypropylene. The elastic modulus $E=1.4 \mathrm{GPa}$. The Poisson's ratio $\mu=0.42$. The allowable stress $\sigma=34 \mathrm{MPa}$. The allowable shear stress $[\tau]=17 \mathrm{MPa}$. Based on the size of the hinges given in Delimont $e t$ al. (2015), Dewalque et al. (2016), and Naves et al. (2017), the parameter values of the hinge are set. The radii of the inner cylinder and outer ring are $r=6 \mathrm{~mm}, R=30 \mathrm{~mm}$. The beam length $L_{1}=20 \mathrm{~mm}, L_{2}=5 \mathrm{~mm}$. The width and thickness of the beam section are $b=2.5 \mathrm{~mm}$ and $h=0.2 \mathrm{~mm}$.

\section{SIMULATION FOR ROTATIONAL STIFFNESS}

In the nonlinear analyses, the stiffness of structures decreases with the applied load. The deformation of the hinge calculated by using linear theory is larger than the deformation calculated by using nonlinear when the load is constant. Therefore, the rotational angle calculated by using linear analyses method can be achieved. At the same time, the stiffness 
model does not contain the nonlinear characteristic of the hinge. To verify the stiffness model of the hinge, the linear analyses were conducted.

The inner cylinder was modeled by solid, which was divided by using hexahedral mesh. The flexible beam was modeled by beam elements. The three displacements in space were constrained and the rotation on $z$ direction was relaxed when $z$-direction torque was applied. When the rotation angle of the inner cylinder was $55^{\circ}$, the stress and deformation of this hinge were analyzed, which are shown in Fig. 8. The maximum von Mises stress is $12 \mathrm{MPa}$. The corresponding moment, checked by finding the reaction force in ANSYS, is $1.58 \mathrm{~N} \cdot \mathrm{mm}$. The rotational stiffness of the hinge is $1.64 \mathrm{~N} \cdot \mathrm{mm} / \mathrm{rad}$. Therefore, the allowable rotation angle can be larger than $55^{\circ}$.

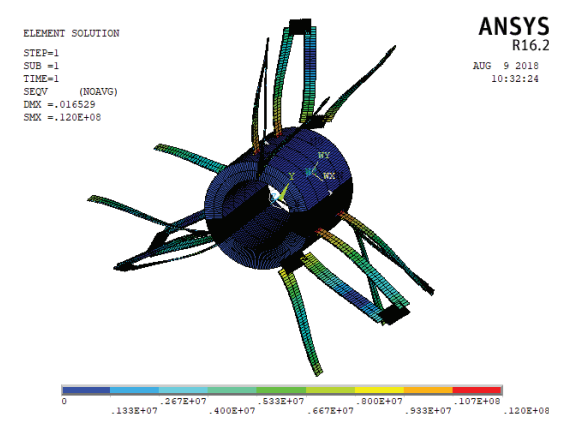

Figure 8. The stress and deformation of the hinge under the $z$-direction torque.

When a set of different torques was applied to the flexible hinge, the corresponding rotation angles were calculated. It can be seen from Fig. 9 that the simulation results are close to the theoretical results when the flexible hinge deformed under the $z$-direction torque. The error is about $1 \%$ to $2 \%$.

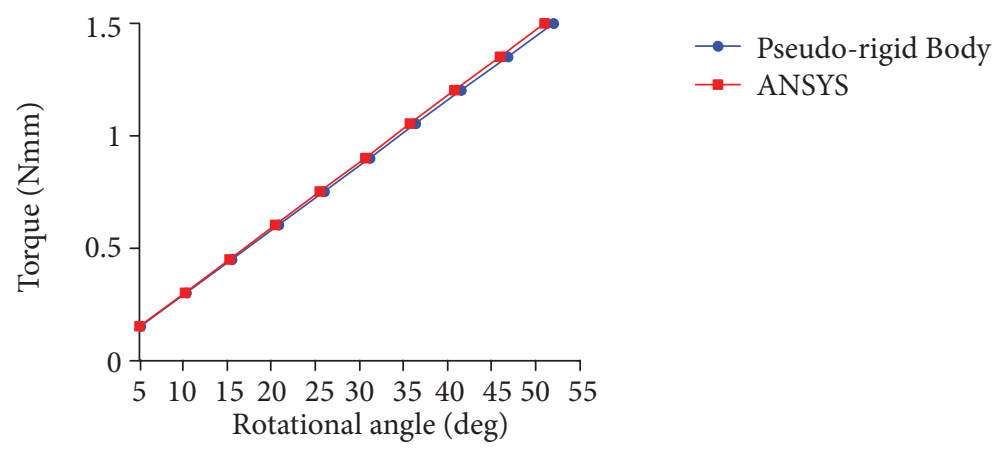

Figure 9. Comparison of simulation and theoretical results.

\section{SIMULATION FOR RADIAL STIFFNESS}

When a force in the $y$ direction was applied on the inner cylinder, the flexible hinge deformed. The displacement of the inner cylinder is in $y$ direction. The stress and displacement were analyzed. The stress and displacement diagram are shown in Fig. 10. When the displacement of the inner cylinder is $1 \mathrm{~mm}$ in vertical direction, the reaction force in this direction is $0.98 \mathrm{~N}$. The radial stiffness of the flexible hinge is $0.98 \mathrm{~N} / \mathrm{mm}$.

Different forces were applied to the hinge to obtain simulated displacement values. The radial stiffness of the theoretical and simulation are $0.98 \mathrm{~N} / \mathrm{mm}$ and $0.99 \mathrm{~N} / \mathrm{mm}$. The accuracy of the stiffness model was verified. 


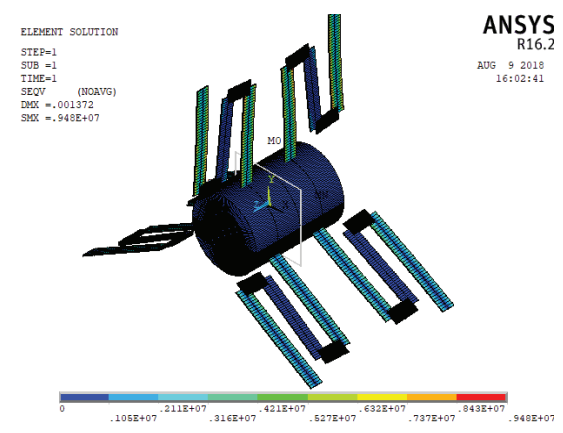

Figure 10. Stress and deformation of the hinge.

\section{STRUCTURE OPTIMIZATIONS OF FLEXIBLE HINGE}

\section{DESIGN VARIABLE}

When the flexible hinge has been determined, the structural dimensions $L_{1}, L_{2}, b$, and $h$ are set to be the design variables (Eq. 23).

$$
X=\left[\begin{array}{llll}
x_{1} & x_{2} & x_{3} & x_{4}
\end{array}\right]^{\mathrm{T}}=\left[\begin{array}{llll}
L_{1} & L_{2} & b & h
\end{array}\right]^{\mathrm{T}}
$$

\section{OBJECTIVE FUNCTION}

The performance of the flexible hinge mainly includes the rotational and radial stiffness. Therefore, an objective function is established to get the rotation capability and radial stiffness of the flexible hinge. The objective function can be given as (Eq. 24)

$$
\operatorname{minimize}\left\{\begin{array}{l}
f_{1}(x) \\
f_{2}(x)
\end{array}\right.
$$

where $f_{1}(x)=\frac{6}{\frac{12\left(x_{1}{ }^{2}+\left(2 x_{1}+r-R\right)^{2}\right)}{k R E x_{3} x_{4}{ }^{3}}+\frac{\left(2 x_{1}+r-R\right) x_{2}}{G R x_{3} x_{4}{ }^{3}\left(1 / 3-0.21 x_{4} / x_{3}\right)}}$, and $f_{2}(x)=-\frac{3 E x_{3} x_{4}}{2\left(\left(x_{1}+\frac{1}{2}\left(2 x_{1}+r-R\right)\right)+\frac{12 x_{2}{ }^{2}}{x_{3}{ }^{2}}\left(x_{1}+x_{2} / 3\right)\right)}$.

\section{RESTRICTIONS}

- $\quad$ Restrictions of the size (Eq. 25)

$$
\left\{\begin{array}{l}
b \leq L_{2} \\
h \leq \mathrm{b} \\
L_{2} / \mathrm{b} \leq 2 \\
3 \leq L_{1} / L_{2}
\end{array}\right.
$$

- $\quad$ Restrictions of boundary (Eq. 26)

$$
\left\{\begin{array}{l}
12 \leq L_{1} \leq 22 \\
2 \leq L_{2} \leq 10 \\
1 \leq b \leq 10 \\
0.2 \leq \mathrm{h} \leq 2
\end{array}\right.
$$


- Performance constraints

Assume that the torque and force applied on the flexure hinges are $M=1.5 \mathrm{~N} \cdot \mathrm{mm}$ and $F=2 \mathrm{~N}$, respectively. The corner marks of the stresses caused by torque and force are "a" and " $b$ ", respectively. The stress of the hinge satisfies (Eq. 27):

$$
\left\{\begin{array}{l}
\sigma_{\mathrm{a}} \leq[\sigma] \\
\sigma_{\mathrm{b}} \leq[\sigma] \\
\tau_{\mathrm{a}} \leq[\sigma] / 2
\end{array}\right.
$$

The optimization of flexible hinge is conducted by MATLAB. The optimization function "fgoalattain" is used to minimize a multi-objective optimization problem. The parameters of the optimization function are set based on the design variable, objective function, and restrictions.

\section{COMPARING THE PERFORMANCE OF THE FLEXIBLE HINGE BEFORE AND AFTER OPTIMIZATION}

The sizes of the rings are given as $r=6 \mathrm{~mm}$ and $R=30 \mathrm{~mm}$. The other dimensions and stiffness before and after multi-objective optimization are shown in Table 1 . The rotational stiffness decreases by $21.8 \%$. At the same time, the radial stiffness increased by $38 \%$. The rotational stiffness significantly decreased, when the radial stiffness significantly increased. After the optimization, because the rotational stiffness of the hinge decreases when the radial stiffness is almost constant, the rotational performance of the flexible hinge is relatively improved.

Table 1. Before and after optimization.

\begin{tabular}{|c|c|c|}
\hline Parameters & Before optimization & \multicolumn{2}{|c|}{ After optimization } \\
\hline$L_{1}(\mathrm{~mm})$ & 20 & 22 \\
\hline$L_{2}(\mathrm{~mm})$ & 5.0 & 4 \\
\hline$b(\mathrm{~mm})$ & 2.5 & 2.2 \\
\hline$h(\mathrm{~mm})$ & 0.2 & 0.2 \\
\hline$K_{\mathrm{R}}(\mathrm{N} \cdot \mathrm{mm} / \mathrm{rad})$ & 1.65 & 1.29 \\
\hline$K_{\mathrm{T}}(\mathrm{N} / \mathrm{mm})$ & 0.99 & 1.37 \\
\hline
\end{tabular}

\section{CONCLUSIONS}

In this paper, the theory of the fixed-guide beam structure in the pseudo-rigid body method and the series-parallel relationship of the flexible beam are used to establish the equivalent rotational stiffness model of flexible hinge. The radial stiffness model of the hinge is established based on deformation energy theory. The finite element analysis is conducted to verify the stiffness models. The finite element analysis in ANSYS validates the correctness of this model.

In order to improve the rotation angle under the premise of ensuring the radial stiffness, an optimized model was established in MATLAB, and the multi-objective optimization algorithm was used to optimize the structural parameters of the flexible hinge. The optimization results show that the rotational stiffness decreases from $1.65 \mathrm{~N} \cdot \mathrm{mm} / \mathrm{rad}$ to $1.29 \mathrm{~N} \cdot \mathrm{mm} / \mathrm{rad}$, the radial stiffness increases from $0.99 \mathrm{~N} / \mathrm{mm}$ to $1.37 \mathrm{~N} / \mathrm{mm}$. The rotational stiffness of the flexible hinge obviously decreases. That is to say the rotation angle and accuracy have been improved by using the optimization method.

\section{ACKNOWLEDGEMENTS}

The work reported was partially performed when the first author was a visiting researcher at KTH Mechanics, Stockholm, Sweden. We would like to thank Prof. Anders Eriksson, Royal Institute of Technology, for his helpful suggestions on this work. 


\section{AUTHOR'S CONTRIBUTION}

Conceptualization, Zhang J and Yan K; Methodology, Yan K; Investigation, Zhang J and Yan K; Writing - Original Draft, Zhang J and Yan K; Writing - Review and Editing, Zhang J and Kou Z; Funding Acquisition, Zhang J; Resources, Kou Z; Supervision, Zhang J.

\section{FUNDING}

National Natural Science Foundation of China-Yunnan Joint Fund [http://dx.doi.org/10.13039/501100011002]

Grant No: JCYJ20170413104438332

National Natural Science Foundation of China [http://dx.doi.org/10.13039/501100001809]

Grant No: 51505319

Shanxi Provincial Higher School Outstanding Innovative Team

Grant No: 2014TD040

Shenzhen Research Funds

Grant No: JCYJ20170413104438332

\section{REFERENCES}

Delimont IL, Magleby SP, Howell LL (2015) A family of dual-segment compliant joints suitable for use as surrogate folds. Journal of Mechanical Design 137(9):092302. https://doi.org/10.1115/1.4030875

Dewalque F, Collette JP, Brüls $\mathrm{O}$ (2016) Mechanical behaviour of tape springs used in the deployment of reflectors around a solar panel. Acta Astronautica 123:271-282. https://doi.org/10.1016/i.actaastro.2016.03.029

Fenci GE, Currie NGR (2017) Deployable Structures Classification: A Review. International Journal of Space Structures 32(2):112-130. https://doi.org/10.1177/0266351117711290

Hopkins JB, Culpepper ML (2011) Synthesis of precision serial flexure systems using freedom and constraint topologies (FACT). Precision Engineering 35(4):638-649. https://doi.org/10.1016/i.precisioneng.2011.04.006

Hopkins JB, Panas RM (2013) Design of flexure-based precision transmission mechanisms using screw theory. Precision Engineering 37(2):299-307. https://doi.org/10.1016/j.precisioneng.2012.09.008

Howell LL (2001) Compliant mechanisms. New York: Wiley-Interscience.

Li B, Wang SM, Yuan R, Xue XZ, Zhi CJ (2016) Dynamic characteristics of planar linear array deployable structure based on scissorlike element with joint clearance using a new mixed contact force model. Proceedings of the Institution of Mechanical Engineers, Part C: Journal of Mechanical Engineering Science 230(18):3161-3174. https://doi.org/10.1177/0954406215607903

Liu PB, Yan P (2017) A modified pseudo-rigid-body modeling approach for compliant mechanisms with fixed-guided beam flexures. Mechanical Sciences 8:359-368. https://doi.org/10.5194/ms-8-359-2017

McGuire T, Hirsch M, Parsons M, Leake S, Straub J (2016) A cubesat deployable solar panel system. Presented at: SPIE Commercial + Scientific and Sensing Imaging; Baltimore, USA. https://doi.org/10.1117/12.2223566

Meguro A, Shintate K, Usui M, Tsujihata A (2009) In-orbit deployment characteristics of large deployable antenna reflector onboard engineering test satellite VIII. Acta Astronautica 65(9-10):1306-1316. https://doi.org/10.1016/i.actaastro.2009.03.052

Midha A, Bapat SG, Mavanthoor A, Chinta V (2015) Analysis of a Fixed-Guided Compliant Beam with an Inflection Point Using the PseudoRigid-Body Model Concept. Journal of Mechanisms and Robotics 7(3):031007. https://doi.org/10.1115/1.4028131

Naves M, Brouwer DM, Aarts RGKM (2017) Building block-based spatial topology synthesis method for large-stroke flexure hinges. Journal of Mechanisms and Robotics 9(4):041006. https://doi.org/10.1115/1.4036223

Qiu LF, Wei ZH, Yu BQ, Wang LH (2014) Analysis of equivalent stiffness and parameter optimization of LET flexure hinge. Engineering Mechanics 31(1):188-192. https://doi.org/10.6052/j.issn.1000-4750.2012.09.0649 
Šalinić S, Nikolić A (2018) A new pseudo-rigid-body model approach for modeling the quasi-static response of planar flexure-hinge mechanisms. Mechanism and Machine Theory 124:150-161. https://doi.org/10.1016/j.mechmachtheory.2018.02.011

Su HJ, Yue C (2013) Type synthesis of freedom and constraint elements for design of flexure mechanisms. Mechanical Sciences 4:263277. https://doi.org/10.5194/ms-4-263-2013

Sun DY, Chen GP (2016) Kinematic accuracy analysis of planar mechanisms with clearance involving random and epistemic uncertainty. European Journal of Mechanics - A/Solids 58:256-261. https://doi.org/10.1016/j.euromechsol.2016.02.007

Sun F, Hopkins JB (2017) Mobility and constraint analysis of interconnected hybrid flexure systems via screw algebra and graph theory. Journal of Mechanisms and Robotics 9(3):031018. https://doi.org/10.1115/1.4035993 